\title{
CAUSALITY AND ANALYTICITY
}

BY

Y. FOURES AND I. E. SEGAL

1. Introduction. We treat an abstract version of a linear mechanical system, demonstrating a connection between causality in the system and analyticity of its gain function. This function is in general merely Lebesgue measurable; it is suitably analytic if and only if the system is causal in the sense that the future cannot influence the past. This result is for the "continuous" case in which the output is globally a continuous function of the input. Systems described by partial differential equations with constant coefficients will not in general be of this type, and we are led thereby to an examination of the influence of causality in the "discontinuous" case.

In strictly mathematical terms, we investigate a special type of unbounded operator on a Hilbert space of functions over a vector group, in which a convex cone is distinguished. All operators are required to commute with group translations, and are then characterized by their gain functions, which are defined on the dual group. We are concerned with the relationship between the following two conditions on an operator: (a) it leaves the manifold of functions vanishing outside the cone invariant, and (b) its gain function can be extended to be (complex) analytic in the "tube" over the dual cone. Our results are complete in the case of bounded operators and also in the case of the Green's operator of a linear partial differential equation with constant coefficients. In the latter case the two conditions are in turn closely related to the condition that the equation be hyperbolic. In general, (a) and (b) imply each other in the presence of simple side conditions which concern the regularity of the operator and the growth of the analytic extension of the gain. In particular, an analytic function $F(z)$ on the tube which grows no more rapidly than $\exp (\|z\| \alpha)$ for some $\alpha<1$ is the analytic extension of the gain of a closed causal operator.

By means of the causal operators we are able to treat domains of dependence for arbitrary continuous linear operators commuting with group translations. For any such operator $T$ there is a unique minimal closed convex set $D$ which acts as the support of $T$ in the sense that if $f$ vanishes outside the compact set $A$, then $T f$ vanishes outside $A \oplus D$.

The conceptual as well as the technical basis of the present work is due primarily to Wiener (cf. $[7 ; 9]$ ), and secondarily to Bochner (cf. $[1 ; 2]$ ), who extended relevant parts of Wiener's work on the Laplace transform from one to $n$ dimensions. Strong use of the Laplace transform in dealing with partial differential equations, along lines related to our own use, is made by Leray

Presented to the Society, December 30, 1953; received by the editors January 29, 1954. 
$[5 ; 6]$, and our result in this connection should be compared with that of Gårding [4], who made some use also of Laplace transforms.

2. Technical preliminaries. For the purposes of the present paper, a (mechanical) system is defined as a mapping $T$ from one class $I$ of functions on a real vector group $G$ to another such class $O$; the elements of $I$ are inputs, those of $O$ outputs. It will be assumed that $G$ is causally oriented, by which is meant that there is given in $G$ a closed convex cone $C$ with nonempty interior. The term cone in the following will always refer to a cone of this particular type. A system (or its operator) is said to be strongly causal if whenever two inputs agree on a set of the form $a \ominus C$ (i.e., intuitively speaking, on the part relative to $a$ ) for some $a$ in $G$, then the corresponding outputs agree on this set. The notation $a \ominus C$ here signifies the set $[a-x: x \in C]$. A system (or its operator) is homogeneous if translation of an input through a given vector involves the same translation of the outputs, i.e. if $I$ and $O$ are invariant under the translations $U_{a}$, where $\left(U_{a} f\right)(x)=f(x+a)$ for any input $f$, and the equality $T U_{a}=U_{a} T$ holds for all $a$ in $G$.

A Hilbert system is one in which both $I$ and $O$ consist of a linear set of square-integrable functions on $G$ to a complex Hilbert space $K$, the value space of the system. All systems considered here will be of this type and it will be further assumed always that the dimension of $K$ is (at most) countable. The space $\mathfrak{T C}$ of all inputs or outputs is itself a pre-Hilbert space with respect to the inner product $(f, g)_{H}=\int_{O}(f(a), g(a))_{K} d a$, where the subscripts " $H$ " and " $K$ " indicate inner products in the respective spaces, and $d a$ is the element of Lebesgue measure on $G$. A system is called continuous or closed respectively if its operator, as an operator on a Hilbert space, is such, where, in the present paper, a continuous operator will be taken to be everywhere defined (as well as bounded) and a closed operator will be taken to be densely defined (as well as to have a closed graph).

A closed homogeneous Hilbert system is characterized by its gain function, this being the function $T(\cdot)$ whose existence is asserted by

THEOREM 1. Let $\Sigma$ be a closed homogeneous Hilbert system with operator $T$, group $G$, and value space $K$. Then there exists a function $T(\cdot)$ on the dual $G^{*}$ of $G$ to the closed operators on $K$, such that if $f$ is in the domain of $T$, and if $F$ denotes the Plancherel transform of $f$, then almost everywhere on $G^{*}, F\left(a^{*}\right)$ is in the domain of $T\left(a^{*}\right)$, and the Plancherel transform of $T f$ is $T(\cdot) F(\cdot)$.

We recall that the Plancherel (or $L_{2}$-Fourier) transform of $f$ is given by the equation

$$
F\left(a^{*}\right) \sim \int_{G} a^{*}(a) f(a) d a,
$$

or more precisely, $F(\cdot)$ is the $L_{2}$-limit of $\left\{\int_{C_{n}} a^{*}(a) f(a) d a\right\}$, where $\left\{C_{n}\right\}$ is an arbitrary monotone increasing sequence of compact sets in $G$ which cover 
$G$. The proof of the theorem goes through successive stages, depending on the properties of $T$, and begins with the case when $T$ is bounded. Assuming this and putting $\Phi$ for the operation of taking the Plancherel transform on $L_{2}(G, K)$ to $L_{2}\left(G^{*}, K\right)$ (where for any subset $E$ of a vector group, $L_{2}(E, K$ ) denotes the class of all square-integrable functions from $E$ to $K$ ), it follows from the ${ }_{i}$ homogeneity of $T$ that $\Phi T \Phi^{-1}$ commutes with all multiplications by characters, $a^{*}(a)$, on $L_{2}\left(G^{*}, K\right)$. It therefore commutes with all operators in the weakly closed ring generated by such multiplications, and so (by the well-known structure of this ring) with all multiplications by bounded measurable functions on $G^{*}$. The most general bounded linear operator of this sort is well known to have the stated form, which is unique (modulo null sets) and has the following properties needed later. For any two such operators, $S$ and $T$, these equations hold a.e.: (1) $(S+T)\left(a^{*}\right)=S\left(a^{*}\right)+T\left(a^{*}\right)$, (2) $(S T)\left(a^{*}\right)=S\left(a^{*}\right) T\left(a^{*}\right),(3) S^{*}\left(a^{*}\right)=S\left(a^{*}\right)^{*}$.

Next suppose that $T$ is hyperhermitian (i.e. $=T^{*}$ ) and set $T \sim \int \lambda d E_{\lambda}$, where the resolution of the identity $\left\{E_{\lambda}\right\}$ is normalized by requiring that $E_{\lambda+0}=E_{\lambda}$. For each rational $\rho$, select a gain function $E_{\rho}(\cdot)$, as is possible by virtue of the preceding paragraph. It follows from the properties listed shortly before that for each fixed $\rho, E_{\rho}\left(a^{*}\right)$ is for almost all $a^{*}$ a projection, and since only countably many $\rho$ are involved here, it is no essential loss of generality to take $E_{\rho}\left(a^{*}\right)$ to be a projection for all (rational) $\rho$. Now for an arbitrary pair of rationals $\rho$ and $\sigma$ with $\rho>\sigma$, it is easily seen that $E_{\rho}\left(a^{*}\right) \geqq E_{\sigma}\left(a^{*}\right)$ a.e., and again it may be assumed that this is the case for all $a^{*}$. Now setting, for arbitrary real $\lambda, E_{\lambda}^{\prime}\left(a^{*}\right)=\bigcap_{\rho>\lambda} E_{\rho}\left(a^{*}\right)$, it is straightforward to verify that for each $a^{*},\left\{E_{\lambda}^{\prime}\left(a^{*}\right) ;-\infty<\lambda<\infty\right\}$ is a normalized resolution of the identity, so that $\int \lambda d E_{\lambda}^{\prime}\left(a^{*}\right)$ determines for each $a^{*}$ a hyperhermitian operator $T^{\prime}\left(a^{*}\right)$ on $K$. It will be now shown that $T^{\prime}(\cdot)$ can serve as the $T(\cdot)$ of the conclusion of the theorem.

Note first that $E_{\lambda}^{\prime}(\cdot)$ is a gain function for $E_{\lambda}$, for all $\lambda$. For if $\left\{\rho_{n}\right\}$ is a sequence of rationals decreasing monotonely to $\lambda$, then $\left\{E_{\rho_{n}}\right\}$ decreases monotonely to $E_{\lambda}$, which implies that $\left\{E_{\rho_{n}}\left(a^{*}\right)\right\}$ decreases monotonely to $\left(E_{\lambda}\left(a^{*}\right)\right.$ ), for almost all $a^{*}$ (using the result, valid for $K$-valued functions by the same proof as for complex-valued ones, that a convergent sequence in $L_{2}(G, K)$ has a subsequence that converges pointwise a.e.). It follows that a.e. $E_{\lambda}^{\prime}\left(a^{*}\right)=E_{\lambda}\left(a^{*}\right)$. From this result it is straightforward to deduce that if $T$ is a finite linear combination of mutually orthogonal projections, then the $T^{\prime}(\bullet)$ obtained above is a gain for $T$. Now if $T$ is bounded, the same result follows by an approximation argument. Specifically, it is no loss of generality to take $T$ to be self-adjoint, say $T \sim \int \lambda d E_{\lambda}$, where $\left\{E_{\lambda}\right\}$ is a normalized resolution of the identity. Let $\left\{\psi_{n}\right\}$ be a sequence of step functions which converges uniformly to the identity function over the interval $[-\|T\|,\|T\|]$, and set $T_{n}=\int \psi_{n}(\lambda) d E_{\lambda}$. By what was proved above, $T_{n}\left(a^{*}\right)$ $=\int \psi_{n}(\lambda) d E_{\lambda}\left(a^{*}\right)$ a.e. For an arbitrary $f$ in $L_{2}(G, K)$, clearly $T_{n} f \rightarrow T f$, so that 
for a suitable subsequence of the integers $\left(n_{i}\right), T_{n_{i}}\left(a^{*}\right) F\left(a^{*}\right) \rightarrow T\left(a^{*}\right) F\left(a^{*}\right)$ a.e. Now $-\|T\| I_{H} \leqq T \leqq\|T\| I_{H}$, and as $A \geqq B$ for bounded self-adjoint $A$ and $B$ if and only if $A-B=C^{2}$, with $C$ bounded and self-adjoint, it follows that a.e. $-\|T\| I_{K} \leqq T\left(a^{*}\right) \leqq\|T\| I_{K}$, i.e. a.e. $\left\|T\left(a^{*}\right)\right\| \leqq\|T\|$. It follows that, a.e., $\left\{T_{n_{i}}\left(a^{*}\right)\right\}$ converges uniformly to $\int \lambda d E_{\lambda}^{\prime}\left(a^{*}\right)$, i.e. to $T^{\prime}\left(a^{*}\right)$, showing that $T^{\prime}(\bullet)$ has the stated property.

For an arbitrary hyperhermitian $T$, set $T_{n}=\int c_{n}(\lambda) d E_{\lambda}$, where $c_{n}$ is the characteristic function of the interval $(-n, n)$, and let $f$ be arbitrary in the domain of $T$. Then $T_{n} f \rightarrow T f$, so that for an appropriate subsequence $\left\{n_{i}\right\}$ of the integers, a.e.

$$
\left[\int c_{n_{i}}(\lambda) d E_{\lambda}^{\prime}\left(a^{*}\right)\right] F\left(a^{*}\right) \rightarrow(\Phi T f)\left(a^{*}\right) .
$$

It follows that, a.e., $F\left(a^{*}\right)$ is in the domain of $T^{\prime}\left(a^{*}\right)$, and that $T^{\prime}(\cdot) F(\cdot)$ is the Plancherel transform of $T f$. Thus $T^{\prime}(\bullet)$ is a gain function for $T$.

Finally let $T$ be an arbitrary closed operator. As is well known, it has a polar form $T=A U$ with $A$ hyperhermitian and $U$ partially isometric, and with both of these operators commutative with every unitary operator with which $T$ is. In the present case this implies that $A$ and $U$ are homogeneous and hence have gains, $A(\cdot)$ and $U(\bullet)$, in accordance with the preceding. Putting $T\left(a^{*}\right)=A\left(a^{*}\right) U\left(a^{*}\right)$, then $T\left(a^{*}\right)$ is a closed operator a.e., as $A\left(a^{*}\right)$ is evidently hypermitian, $U\left(a^{*}\right)$ is bounded a.e., and the product of a hyperhermitian with a bounded operator is closed. Now if $f$ is in the domain of $T$, then $(\Phi T f)\left(a^{*}\right)=(\Phi A U f)\left(a^{*}\right)=A\left(a^{*}\right)(\Phi U f)\left(a^{*}\right)$ a.e. $=A\left(a^{*}\right) U\left(a^{*}\right) F\left(a^{*}\right)$ a.e.

COROLlary 1.1. If $T$ is normal, its gain function $T(\cdot)$ can be assumed to have normal values and to have the property that for any Baire function $\psi, \psi(T)$ has the gain $\psi(T(\cdot))$. With these conditions the gain of $T$ is unique.

It suffices to consider the case when $T$ is hyperhermitian, as the normal case then follows from examination of the real and imaginary parts of $T$. Let $\mathcal{C}$ be the class of all bounded Baire functions $\psi$ for which the stated conclusion concerning $\psi$ is valid. By an argument similar to the one used above in obtaining the existence of a gain function for (hyperhermitian) $T, \mathcal{C}$ is closed under bounded pointwise convergence. From the construction of $T(\bullet)$, it is clear that $\mathcal{C}$ contains'all step functions. It results that $\mathcal{C}$ consists of all bounded Baire functions. By another use of an argument similar to the one mentioned the conclusion of the corollary is proved for arbitrary $\psi$.

To show uniqueness, let $T_{1}(\cdot)$ and $T_{2}(\cdot)$ be gains for $T$ satisfying the given conditions. Then $c_{n}\left(T_{1}(\cdot)\right)$ and $c_{n}\left(T_{2}(\cdot)\right)$ are gains for the bounded operator $c_{n}(T)$, so that a.e. $c_{n}\left(T_{1}\left(a^{*}\right)\right)=c_{n}\left(T_{2}\left(a^{*}\right)\right)$. It is easily deduced from this that $T_{1}\left(a^{*}\right)=T_{2}\left(a^{*}\right)$ a.e.

EXAMPLE 1. Let $T$ be the closure of a partial differential operator involving $m+n$ independent variables $x_{1}, \cdots, x_{m}$ and $y_{1}, \cdots, y_{n}$, and with 
coefficients that are independent of $x_{1}, \cdots, x_{m}$. The gain of $T$, relative to the vector group of all $\left(x_{1}, x_{2}, \cdots, x_{m}\right)$, is a function of the $m$ variables $x_{1}^{\prime}, x_{2}^{\prime}, \cdots, x_{m}^{\prime}$ dual to the $x_{1}, x_{2}, \cdots, x_{m}$, with values in the closed operators on the space of square-integrable functions of $y_{1}, y_{2}, \cdots, y_{n}$.

EXAMPLE 2. If $T$ is a convolution operator on $L_{2}(-\infty, \infty), f \rightarrow f * k$, with $k$ in $L_{1}(-\infty, \infty)$, the gain of $T$ is the Fourier transform of $k$. It is clear from the existence of the Laplace transform that if $k$ vanishes for negative values, i.e. in intuitive terms $T f$ at any "time" depends only on the "past" of $f$, then the gain can be extended to a half-plane so as to be analytic and bounded.

3. Continuous systems. In this section it is shown that the influence of an assumption of causality on an arbitrary continuous homogeneous system is essentially the same as in the special case just described. The converse is also valid.

Theorem 2. A continuous homogeneous system is causal with respect to a cone if and only if its gain admits a bounded analytic extension to the tube over the dual of the cone.

We recall and introduce some pertinent definitions. The dual of a cone $C$ in a vector group $G$ is the cone $C^{*}$ in the dual $G^{*}$ of $G$ consisting of the vectors $x^{*}$ in $G^{*}$ such that $x^{*}(x) \geqq 0$ for all $x$ in $C$. The tube $\Gamma$ over a set $D$ in $G$ is the set of all complex vectors of the form $x+i y$ with $x$ in $D$ and $y$ in $G^{*}$. The spine of $\Gamma$ is the subset $i G^{*}$ (when the origin is in $D$ ). The spine is then canonically isomorphic with $G^{*}$, and via this isomorphism the gain of the system determines a function on the spine. It is convenient to call this latter function the gain also, it being either immaterial or clear from the context in the following which version of the gain is meant. A function $\phi$ on the interior $\Gamma^{0}$ of $\Gamma$ is said to extend a function $\phi_{0}$ on the spine, or to have the boundary values $\phi_{0}$ on the spine, if $\lim _{x \rightarrow 0}^{*} \phi_{x}=\phi_{0}$, where: $\phi_{x}$ is the function on the spine given by the equation $\phi_{x}(i y)=\phi(x+i y) ; x \rightarrow 0$ through values in $C$; and $\lim ^{*}$ denotes the star-limit relative to Lebesgue measure on $G^{*}$, i.e. a function such that every subsequence has again a subsequence converging a.e. to it. An operator-valued function $\phi$ is said to be analytic if $(\phi(z) f, g)$ is a (complex) analytic function in the usual sense, for all vectors $f$ and $g$ in the Hilbert space on which the operators act.

A Hilbert system (or its operator) is said to be causal with respect to a cone $C$ if: (1) whenever an input vanishes except on a set of the form $a \oplus C$, then so also does the corresponding output; (2) the collection of all such inputs spans $L_{2}(a \oplus C)$. While condition (2) is automatic in the case of a continuous system, it is essential in the case of a closed system, as otherwise condition (1) may be trivially satisfied. It is easily seen that a strongly causal operator is necessarily causal. From a general mathematical viewpoint causality appears to be a more useful notion than strong causality, but for continuous systems the two concepts are equivalent. A closed homogeneous 
causal operator in $L_{2}(G)$ is easily seen to be the closure of its restriction to the subset of its domain consisting of those inputs that vanish on a set of the form $a \ominus C$, for some $a$ in $G$, and so in a sense does not depend on the infinite past.

Lemma 2.1. A continuous Hilbert system is strongly causal if and only if it is causal.

Proof. For the "if" part it must be shown that if $f$ vanishes on $a \ominus C$, then so also does $T f$. Putting $C^{0}$ for the interior of $C$, it is obvious that the complement $D$ of $a \ominus C$ in $G$ is covered by the $x \oplus C^{0}$, with $x$ in $D$. By Lindelöf's theorem there is a countable covering of $D$ by the $x \oplus C^{0}$. It follows readily that there exists a sequence $\left\{f_{n}\right\}$ in $\mathfrak{H}$, each member of which vanishes outside a finite union of the $x \oplus C^{0}$, and which converges to the given function $f$. Now if $f_{n}$ vanishes outside $U_{i}\left(x_{i} \oplus C^{0}\right), f_{n}$ can be put in the form $f_{n}=\sum_{i} g_{i}$, with $g_{i}$ vanishing outside $x_{i}+C^{0}$ and square-integrable. Since $T f=\sum_{i} T g_{i}$ and $T g_{i}$ vanishes a.e. outside $x_{i} \oplus C^{0}$ by causality (the boundary of $x_{i} \oplus C^{0}$ having measure zero because of the convexity of $\left.x_{i}+C^{0}\right)$, it follows that $T f_{n}$ vanishes outside of $U_{i}\left(x_{i} \oplus C^{0}\right)$. Hence $T f_{n}$ vanishes outside of $D$, and from the continuity of $T$ it results that $T f$ vanishes outside $D$. The proof of the "only if" part is easy and is omitted.

Proof of theorem. Let $f$ be an input and vanish outside $C$; for the "if" part of the theorem it must be shown that $T f$ also vanishes outside $C$. Let $F$ be the Laplace transform of $f$, i.e. the function on the tube $\Gamma^{0}$ over $C^{* 0}$ given by the equation

$$
F(z)=\int_{C} \exp (-z \cdot a) f(a) d a,
$$

where we write $z \cdot a$ instead of $z(a)$ and uniquely define $z(a)$ for complex vectors $z$ by requiring linearity in $z$. Let $\phi$ be the bounded analytic extension to the tube $\Gamma^{0}$ over $C^{* 0}$ of the gain $\phi_{0}$ of $T$. Let $\mathfrak{F C}^{\prime}$ be the collection of all $K$-valued analytic functions $L$ on $\Gamma^{0}$ such that $\int_{\sigma^{*}}\|L(x+i y)\|^{2} d y$ is uniformly bounded for all $x$ in $C^{* 0}$, where a $K$-valued function $L$ is analytic if $(L(\cdot), k)$ is for every $k$ in $K$ a complex-valued analytic function in the usual sense. (The measurability of $\|L(x+i y)\|$ results from the separability of $K$ together with the formula $\|L(z)\|=\sup |(L(z), k)|$, the sup taken over all $k$ in the unit sphere of $K$.) It is well-known for the case that $K$ is 1-dimensional that $\mathfrak{H C}^{\prime}$ consists of all the Laplace transforms of inputs that vanish outside $C$, and it is readily deduced from this that the same is true for arbitrary $K$.

By expansion into an orthonormal basis it can be seen that $\phi(z) F(z)$ is an analytic function on $\Gamma^{0}$, from which it follows that it is in $\mathcal{F C}^{\prime}$. It is therefore the Laplace transform of an element $g$ of $\mathfrak{F}$ which vanishes outside $C$.

A simple computation based on the Plancherel theorem shows that the Laplace transform of $g$ has boundary values on the spine in the sense of Hilbert 
space convergence, and hence also in the sense defined above involving star convergence (the usual proof that mean convergence implies star convergence applies equally well to $K$-valued functions) which coincide a.e. with the Plancherel transform of $g$ (with the canonical identification of the spine of $\Gamma$ with $\left.G^{*}\right)$. By the same token the boundary values of $F$ are given by the Plancherel transform of $f$. Thus $F_{x}$ (where $F_{x}(i y)=F(x+i y)$ ) is star convergent to $F_{0}, \phi_{x}$ is star convergent to $\phi_{0}$, and $\phi_{x}(\cdot) F_{x}(\bullet)$ is star convergent to the Plancherel transform of $g$. It results that this transform is $\phi_{0}(\cdot) F_{0}(\cdot)$, i.e. $g=T f$. In particular, $T f$ vanishes outside $C$.

In presenting the "only if" part of the proof, we assume to begin with that $K$ is 1 -dimensional. Let the operator $T$ of the system be causal, so that as noted above it leaves $L_{2}(C)$ invariant. For $f$ in $L_{1}(G)$, let $A_{f}$ be the operator on the Hilbert space $\mathfrak{H C}$ of all inputs given by the equation $A_{f_{g}}=f * g$,

$$
(f * g)(a)=\int_{G} f(b) g\left(b^{-1} a\right) d b ;
$$

it is well known that $A_{f}$ is bounded and is in the weakly closed ring generated by translations by the elements of $G$. Now if $f$ and $g$ are both in $L_{1}(C) \cap L_{2}(C)$, then $T(f * g)=T A_{f o}=A_{f} T_{\theta}=f * T_{\theta}$ which by symmetry must also equal $g * T_{f}$. Denoting the operation of taking the Laplace transform by $\mathcal{L}$, it follows that $\mathcal{L}(f) \mathcal{L}(T g)=\mathcal{L}(g) \mathcal{L}(T f)$. It results that the function $\mathcal{L}(T f) / \mathcal{L}(f)$, which is well-defined and analytic at all points of $\Gamma^{0}$ at which $\mathcal{L}(f)$ does not vanish, is independent of $f$ in $L_{1}(C) \cap L_{2}(C)$. As for any point $z_{0}$ in $\Gamma^{0}$ there is such an $f$ whose Laplace transform does not vanish at $z_{0}$ (otherwise $\exp \left(-z_{0} \cdot a\right)$ would give an element of $L_{2}(C)$ orthogonal to all such $f$, contradicting the density of $L_{1}(C) \cap L_{2}(C)$ in $\left.L_{2}(C)\right)$, it can be concluded that there exists a function $\phi$ analytic everywhere in $\Gamma^{0}$ and such that $\mathcal{L}(T f)(z)=\phi(z)(\mathcal{L} f)(z)$ for all $f$ in $L_{1}(C)$ or $L_{2}(C)$ and all $z$ in $\Gamma^{0}$. The equation holds actually for all $f$ in $L_{2}(C)$, since the value of the Laplace transform of $f$ depends continuously on $f$, relative to the $L_{2}$-metric. By examining the boundary values of $\phi(\cdot)(\mathcal{L} f)(\cdot)$ on the spine as in the first part of the proof it can be deduced that $\phi$ has boundary values on the spine which agree with the gain of $T$.

For the case that $K$ is 1 -dimensional, it remains only to show that $\psi$ is bounded in $\Gamma^{0}$. For this purpose we require the following result, whose proof uses a smoothing method also employed in [3] and [9].

LeMmA 2.2. If $F$ is analytic on $\Gamma^{0}$ and $\int a^{*}|F(x+i y)|^{2} d y$ is (uniformly) bounded for $x$ in $C^{0}$, then for $z \in \Gamma^{0}$

$$
|F(z)| \leqq \underset{y \in \sigma^{*}}{\operatorname{esssup}}|F(i y)| \text {. }
$$

When $F$ is bounded in $\Gamma^{0}$ the result is known (cf. [9]). For a general $F$, form $F_{N}(z)=\int G^{*} F(z+i v) \psi_{N}(v) d v$, where $\psi_{N}$ is proportional to the characteristic function of the compact neighborhood $N$ of the origin in $G^{*}$, via a con- 
stant chosen so that $\int_{G^{*}} \psi_{N}(y) d y=1$. Then it is easily seen that $F_{N}$ has the same properties as $F$, and is bounded (by $\left\|\psi_{N}\right\|_{2} \cdot \sup _{x}\left(\int_{G^{*}}|F(x+i y)|^{2} d y\right)^{1 / 2}$ ), so that for $z \in \Gamma^{0}$,

$$
\left|F_{N}(z)\right| \leqq \underset{v \in G^{*}}{\operatorname{ess} \sup }\left|F_{N}(i y)\right| .
$$

Choosing a sequence of $N$ 's with intersection equal to the origin, and taking the limit superior on both sides of the preceding inequality, it is easy to conclude that $|F(z)| \leqq \operatorname{ess} \sup _{\nu \in \sigma^{*}}|F(i y)|$.

Completion of proof. Let $\epsilon_{1}, \epsilon_{2}, \cdots, \epsilon_{n}$ be $n$ linearily independent vectors in $C$, where $n$ is the dimension of $G$, and set

$$
F(z, \epsilon)=\prod_{k=1}^{n}\left(1+\epsilon_{k} \cdot z\right)^{-1}
$$

where $\epsilon$ stands for the set of vectors $\epsilon_{1}, \epsilon_{2}, \cdots, \epsilon_{n}$. It is easily seen that $F(\cdot, \epsilon)$ is in the class $\mathfrak{H}_{2}$ of functions analytic in $\Gamma^{0}$ with $\int_{G}|F(x+i y)|^{2} d y$ uniformly bounded for $x$ in $C^{* 0}$. It follows from Lemma 2.2 that

$$
|F(z, \epsilon) \phi(z)| \leqq \underset{v \in \sigma^{*}}{\operatorname{ess} \sup }|F(i y, \epsilon) \phi(i y)|,
$$

so that as $\left|1+i \epsilon_{k} \cdot y\right| \geqq 1$

$$
|F(z, \epsilon) \phi(z)| \leqq \underset{\nu \in G^{*}}{\operatorname{ess} \sup }|\phi(i y)| .
$$

Letting $\epsilon \rightarrow 0$, it results that $\phi(z)$ is bounded for $z$ in $\Gamma^{0}$ by $\|T\|$.

Now let $K$ be $n$-dimensional. Let $x$ and $y$ be arbitrary in $K$, let $f$ be arbitrary in $L_{2}(G)$, and set $g=(T(x \otimes f), y)$, so that $g$ is a complex-valued function on $G$ well-defined modulo null sets. Now $g$ is in $L_{2}(G)$ for putting $h=T(x \otimes f)$ and expanding $h$ in the orthogonal series $h=\sum_{i} h_{i} f_{i}$, where $\left\{f_{i}\right\}$ is a maximal orthonormal set in $L_{2}(G)$ and the $h_{i}$ are in $K$ (but not necessarily orthogonal), $g=(h, y)=\sum\left(h_{i}, y\right) f_{i}$. Thus $\|g\|_{2}^{2}=\sum_{i}\left|\left(h_{i}, y\right)\right|^{2} \leqq\|y\|^{2} \sum_{i}\left\|h_{i}\right\|^{2}=\|y\|^{2}\|h\|^{2}$. The mapping $T_{x, y}: f \rightarrow g$ is thus a linear transformation on $L_{2}(G)$, and the foregoing estimates show that $\left\|T_{x, y}\right\| \leqq\|T\|\|x\|\|y\|$. Since $T_{x, y}$ is obviously causal, it follows from the case in which $K$ is one-dimensional that $T_{x, y}$ has a Laplace transform $\Phi_{x, y}(z)$ (by which we shall mean the extension of its gain to the tube over the dual cone) with bound not greater than $\|T\|\|x\|\|y\|$. As $\Phi_{x, y}(z)$ is a bounded form in $x$ and $y$, it follows that there exists a unique bounded linear operator $\phi(z)$ such that $\Phi_{x, y}(z)=(\phi(z) x, y)$, and this function $\phi$ clearly is the bounded analytic extension of the gain whose existence was asserted.

4. Closed systems. In dealing with the case of unbounded homogeneous operators, it will be assumed that the space $K$ of input values is one-dimensional, in order to avoid extraneous complications. In this case a closed homogeneous operator is necessarily normal. In this section it is shown that 
if an operator is causal, its gain tends to have an analytic extension to the tube over the dual cone. The main contents, however, are to the effect that an analytic function on this tube which does not grow too rapidly is the extension of the gain of some such regularly causal operator. It is clear that some restriction on the growth of the function is necessary, e.g. the function $e^{z}$ in the region $R(z) \geqq 0$ is the extension of the gain of the noncausal operator $f(x) \rightarrow f(x+1)$. While our condition for an analytic function to be of the stated type is probably not necessary, it is best possible of its kind and shows, for example, that any analytic function in the region $R(z) \geqq 0$ which grows no faster than $e^{|z|^{\alpha}}$ for some $\alpha<1$ is of this type.

THEOREM 3. The gain of a closed homogeneous causal operator, whose domain contains at least one nonzero integrable function vanishing outside the causal cone( $\left.{ }^{1}\right)$, admits an analytic extension to the tube over the dual cone.

Conversely, if $\phi$ is analytic in the open tube over a proper cone $C^{*}$, then $\phi$ is the extension of the gain of a closed homogeneous operator that is causal with respect to the dual cone $C$, provided $|\phi(z)| \leqq \mu e^{|| z||^{\alpha}}$, where $\mu$ and $\alpha$ are constants with $\alpha<1$, and $\|z\|$ is the usual unitary norm in $n$ complex dimensions.

Let $T$ be causal with respect to the cone $C$ as well as closed and homogeneous. If $f$ is nonzero but otherwise arbitrary in $L_{2}(C) \cap \mathcal{D}_{T}$, then putting $\mathcal{L}$ for the operation of taking the Laplace transform, $\phi=\mathcal{L}(T f) / \mathcal{L}(f)$ is readily seen to be a meromorphic extension of the gain of $T$. Now if $g$ is a nonzero element of $L_{1}(C) \cap D_{T}$, then conventional Fourier analysis shows that $T f * g=f * T g$, whence $\mathcal{L}(T f) \mathcal{L}(g)=\mathcal{L}(f) \mathcal{L}(T g)$. It results that $\mathcal{L}(T f) / \mathcal{L}(f)$ $=\mathcal{L}(T g) / \mathcal{L}(g)$, so that all the $\mathcal{L}(T f) / \mathcal{L}(f)$ are the same analytic function. As $L_{2}(C) \cap \mathcal{D}_{T}$ is dense in $L_{2}(C)$, for any $z$ in the tube over $C$ there exists an element $f$ of $L_{2}(C) \cap \mathcal{D}_{T}$ such that $\mathcal{L}(f)(z) \neq 0$, as otherwise $e^{-z \cdot u}$, as a function of $u$, would be orthogonal to $L_{2}(C) \cap \mathcal{D}_{T}$. Hence $\phi$ is analytic throughout $\Gamma^{0}$.

To prove the converse we employ a series of lemmas. Throughout these, $C^{*}$ is a given proper cone; $\phi$ is a given function analytic in the open tube $\Gamma^{0}$ over $C^{*}$ and satisfying the inequality $|\phi(z)| \leqq \mu e^{\| z||^{\alpha}}$ throughout $\Gamma^{0}$; $e_{1}^{*}, \cdots, e_{n}^{*}$ is a fixed basis in the dual $G^{*}$ of $G ;\left(x_{1}, \cdots, x_{n}\right)$ are coordinates in $G^{*}$ with respect to this basis while $\left(z_{1}, \cdots, z_{n}\right)$ are coordinates in $G^{*}+i G^{*}$ (the complex linear extension of $G^{*}$ ) relative to the same basis. $\mathfrak{H C}_{2}\left(C^{*}\right)$ denotes the space of all analytic functions $F$ over $\Gamma^{0}$ for which

is finite.

$$
\|F\|^{2}=\sup _{x \in C^{* 0}} \int_{G^{*}}|F(x+i y)|^{2} d y
$$

(1) This assumption is probably unnecessary, but it is satisfied by all specific reasonable operators, and its use eliminates the need for a close examination at this point of the boundary values of analytic functions of several complex variables. When $G$ is one-dimensional, the lack of necessity of the assumption follows from the fact that an analytic function in a circle is determined by its boundary values on any arc. 
Lemma 3.1. Let $C^{*}$ be contained in the first octant $\left[x \in G^{*}: x_{i} \geqq 0\right.$; $i=1,2, \cdots, n]$, and set $F(z)=\prod_{j=1}^{n} \exp \left(-z_{j}^{\beta}\right)$, with $\beta$ a fixed number satisfying the inequality $\alpha<\beta<1$. Then $\phi F$ is in $\mathfrak{F}_{2}\left(C^{*}\right)$.

As $\phi F$ is analytic in the open tube over the first octant, it is only necessary to verify the finiteness of the norm of $\phi F$. Now

$$
\int_{G^{*}}|(\phi F)(x+i y)|^{2} d y \leqq \mu^{2} \int_{G^{*}} \prod_{j=1}^{n} \mid \exp \left(-2 z_{j}^{\beta}\right) \exp \left(2\left|z_{j}\right|^{\alpha}\right) d y
$$

which shows that is is sufficient to prove the uniform boundedness for $x>0$ of

$$
\int_{-\infty}^{\infty} \mid \exp \left\{-2(x+i y)^{\beta}\right\} \exp \left\{2|x+i y|^{\alpha}\right\} d y .
$$

Putting $x+i y=r e^{i \theta},\left|\exp \left\{-2(x+i y)^{\beta}\right\}\right|=\exp \left\{-2 r^{\beta} \cos \beta \theta\right\} \leqq \exp \left\{-2 u r^{\beta}\right\}$, where $u=\cos \beta \pi / 2$, so it suffices in turn to show the uniform boundedness of $I(x)=\int_{-\infty}^{\infty} \exp \left\{2 r^{\alpha}-2 u r^{\beta}\right\} d y$ for $x>0$.

Let $\epsilon$ be a fixed positive number less than $u$, and let $x_{0}$ be such that $2 r^{\alpha}<2 \epsilon r^{\beta}$, it being easily seen that such an $x_{0}$ exists. Now for $x>x_{0}, I(x)$ $\leqq \int_{-\infty}^{\infty} \exp \left(-\sigma r^{\beta}\right) d y$, with $\sigma$ a positive constant, and as $\exp \left(-\sigma r^{\beta}\right)$ is monotone decreasing in $x$, it results that $I(x) \leqq\left[\int_{-\infty}^{\infty} \exp \left(-\sigma r^{\beta}\right) d y\right]_{x=x_{0}}$ for $x \geqq x_{0}$. On the other hand, for $0<x \leqq x_{0}$, it is clear that

$$
I(x) \leqq \int_{-\infty}^{\infty} \exp \left\{2\left|x_{0}+i y\right|^{\alpha}-2 u|y|^{\beta}\right\} d y<\infty,
$$

and so is uniformly bounded.

LEMмA 3.2. $\phi$ has boundary values $\phi(i y)$ on the spine such that

$$
\int_{K^{*}}|\phi(x+i y)-\phi(i y)|^{2} d y \rightarrow 0
$$

as $x \rightarrow 0$ through values in $C^{*}$, for any compact subset $K^{*}$ of $G^{*}$.

For any function $\psi$ on $\Gamma^{0}$, let $\psi_{x}$ denote the function on $G^{*}$ given by the equation $\psi_{x}(i y)=\psi(x+i y)$. Now $\phi_{x} F_{x}$ is convergent in $L_{2}\left(G^{*}\right)$, as $x \rightarrow 0$ through values in $C^{*}$, where $F$ is the function of the preceding lemma, and $F_{x}$ is convergent uniformly on every compact set to nonvanishing values. Taking quotients, it follows that $\phi_{x}$ converges to a function $\phi_{0}$ on $i G^{*}$ in the fashion indicated.

The next lemma shows that the closed homogeneous operator $T$ with gain $\phi_{0}$ is such that $\mathscr{D}_{T} \cap L_{2}(C)$ is dense in $L_{2}(C)$, and that moreover there is a dense subset of $\mathscr{D}_{T} \cap L_{2}(C)$ that is taken into $L_{2}(C)$ by $T$. This by no means establishes the causality of $T$, however, and in fact the remaining lemmas are required to complete the proof. 
Lemma 3.3. Suppose $C^{*}$ is contained in the first octant. Let $\gamma$ be a real number satisfying the inequality $0<\gamma<1$, and let $\mathcal{D}_{\gamma}$ be the collection of all elements $\mathrm{F}$ of $\mathrm{H}_{2}\left(C^{*}\right)$ with the property that

$$
\int|F(z)|^{2} \exp (2\|z\| \gamma) d y
$$

is uniformly bounded for $x$ in $C^{* 0}$. Then $\mathcal{D}_{\gamma}$ is dense in $\mathfrak{F C}_{2}\left(C^{*}\right)$.

If $F \in \mathcal{D}_{\gamma}$, then also $F_{u} \in \mathcal{D}_{\gamma}$ for all $u$ in $G^{*}$, where $F_{u}(z)=F(z+i u)$, for $\int\left|F_{u}(z)\right|^{2} \exp (2\|z\| \gamma) d y=\int|F(z)|^{2} \exp (2\|z-i u\| \gamma) d y \leqq \int|F(z)|^{2} \exp (2\|z\| r$ $+2\|u\| r) d y$.

It follows, taking the inverse Laplace transform, that the collection $\varepsilon_{\gamma}$ of all such transforms of elements of $\mathcal{D}_{\gamma}$ is invariant under the operation of multiplication by the function of $x(\in G), e^{i u \cdot x}$, for all $u$ in $G^{*}$. This implies that the closure $\varepsilon_{\gamma}^{-}$of $\varepsilon_{\gamma}$ has the form $L_{2}(E)$ for some measurable subset $E$ of $C$, for it is well known that the weakly closed ring generated by all such multiplications on $L_{2}(G)$ consists of all multiplications by bounded measurable functions. The invariance of $\varepsilon_{\gamma}$ under the multiplications by $e^{i u \cdot x}$ implies the same invariance for $\varepsilon_{\gamma}^{-}$, which in turn implies that the projection $P$ on $L_{2}(G)$ with range $\varepsilon_{\gamma}^{-}$commutes with the multiplication algebra of $G$ (=algebra of all multiplications by bounded measurable functions). As this algebra is maximal abelian in the algebra of all bounded linear transformations on $L_{2}(G)$, it results that $P$ is itself the operation of multiplication by some bounded measurable function. As $P$ is a projection, this function is necessarily the characteristic function of some measurable set $E$, which means that $\varepsilon_{\gamma}^{-}=L_{2}(E)$.

Now $\mathscr{D}_{\gamma}$ is invariant under the transformation $F(z) \rightarrow F(z) e^{-a \cdot z}$ for $a$ in $C$, since the $\left|e^{-a \cdot z}\right| \leqq 1$. This implies that $\varepsilon_{\gamma}$, and hence also $\varepsilon_{\gamma}^{-}$, are invariant under the transformations $f(x) \rightarrow f(x-a), a \in C$. It follows that $E$ is invariant, modulo the null sets, under the transformations $x \rightarrow x+a, a \in C$. To show that $E=C$ (modulo the null sets), and thereby complete the proof of the lemma, it now suffices to show that there exists a sequence $\left\{x_{n}\right\}$ in $C^{0}$ such that $x_{n} \rightarrow 0$ and with each $x_{n}$ having an open neighborhood $N_{n}$ that is contained in $E$. For if $x$ is arbitrary in $C^{0}$, then $x-x_{n}$ is in $C^{0}$ for large $n$, so that $x \in x_{n} \oplus C^{0}$, i.e. $C^{0} \subset U_{n}\left(x_{n} \oplus C^{0}\right)$. Now $x_{n} \oplus C^{0}$ is covered by the $a \oplus N_{n}$ with $a$ in $C^{0}$, and so, by Lindelöf's theorem, by the $\left\{a_{n k} \oplus N_{n} ; k, n=1,2, \cdots\right\}$, with the $a_{n k}$ in $C^{0}$. But by what has just been shown, if $E \supset N_{n}$ (modulo null sets), then also $E \supset a_{n k} \oplus N_{n}$ (modulo null sets) for all $n, k$, and hence $E \supset U_{n, k}\left(a_{n k} \oplus N_{n}\right)=C^{0}$ (modulo null sets).

To show the existence of a sequence $\left\{x_{n}\right\}$ and corresponding neighborhoods $\left\{N_{n}\right\}$ with stated properties, note that the boundary values on $i G^{*}$ of the function $F(z)=\prod_{j=1}^{n} \exp \left(-z_{j}^{\beta}\right)$ define an integrable function on $G^{*}$. It follows that the inverse Laplace transform $f$ of $F$ can be taken to be continu- 
ous everywhere. As $F \in \mathcal{D}_{\gamma}$ by Lemma $3.1, f \in \mathcal{E}_{\gamma}$, so that if $f(x) \neq 0$ for some $x$, then some neighborhood $N$ of $x$ is contained in $E$ (modulo null sets). Hence it suffices to show that $f\left(x_{n}\right) \neq 0$ for some sequence $\left\{x_{n}\right\}$ with $x_{n} \rightarrow 0, x_{n} \in C^{0}$, i.e. that $f$ vanishes in no neighborhood $U$ of the origin.

Employing an indirect argument, suppose that $f$ vanishes in some such neighborhood. As $f$ has the form $f(x)=\prod_{j=1}^{n} g\left(x_{j}\right)$, where $g$ is the (1-dimensional) inverse Laplace transform of $e^{-z^{\beta}}$, it results that $g$ vanishes in some neighborhood of zero, say on $[-\epsilon, \epsilon]$. Hence the function $g_{\epsilon}$ given by the equation $g_{\epsilon}(x)=g(x+\epsilon)$ vanishes for $x<0$, so that its Laplace transform is in $\mathcal{F C}_{2}(0, \infty)$. This transform is $e^{e z-z^{\beta}}$, so the following expression must be bounded for $x>0$ :

$$
I(x)=\int_{-\infty}^{\infty}\left|e^{\varepsilon z-z^{\beta}}\right|^{2} d y .
$$

On the other hand, putting $r=|z|$,

$$
I(x) \geqq \int_{-\infty}^{\infty} \exp \left\{2 \epsilon x-2 r^{\beta}\right\} d y=2 e^{2 \epsilon x} \int_{0}^{\infty} e^{-2 r^{\beta}} d y \geqq 2 e^{2 e x} \int_{x}^{\infty} e^{-2 r^{\beta}} d r .
$$

Now if $x>1, \int_{x}^{\infty} e^{-2 r^{\beta}} d r>\int_{x}^{\infty} r^{\beta-1} e^{-2 r^{\beta}} d r=(2 \beta)^{-1} e^{-2 x^{\beta}}$, so that

$$
I(x) \geqq \beta^{-1} \exp \left\{2 \epsilon x-2 x^{\beta}\right\},
$$

which is unbounded as $x \rightarrow \infty$.

The next lemma extends results of Paley and Wiener, notably from the case of functions analytic in a closed half-plane to that of functions analytic in an open half-plane. Part of its proof is a simplification of that of Paley and Wiener.

LemMa 3.4. Let $F$ be a function analytic in the open half-plane $R(z)>0$, and with square-integrable boundary values in the sense of local $L_{1}$-convergence on the imaginary axis. Suppose that $|F(z)| \leqq(\mu+|K(z)|) P(z)$, where $K \in \mathcal{H C}_{2}(0, \infty)$ and $\lim _{r \rightarrow \infty} r^{-1}\left|\log P\left(r e^{i \theta}\right)\right|=0$ uniformly in $\theta,|\theta|<\pi / 2$. Then $F \in \mathfrak{H}_{2}(0, \infty)$.

To be perfectly explicit about the assumption on the boundary values of $F$, it is assumed that $F$ admits an extension to the closed half-plane such that $F(i y)$ is Lebesgue-measurable as a function of $y, \int_{-\infty}^{\infty}|F(i y)|^{2} d y$ is finite, and $\int_{\alpha}^{\beta}|F(x+i y)-F(i y)| d y \rightarrow 0$ as $x \rightarrow 0$ through positive values, for all finite $\alpha$ and $\beta$.

Now let $f$ be the inverse Plancherel transform of the function $F(i y)$, and put $F_{+}$and $F_{-}$for the Laplace transforms of $f_{+}$and $f_{-}$, respectively, where $f_{+}=f c$ and $f_{-}=f(1-c), c$ being the characteristic function of $(0, \infty)$. Now $F_{+}(z)$ is analytic for $R(z)>0$ and has boundary values in the sense of local $L_{1}$-convergence on the imaginary axis, while $F_{-}$is analytic for $R(z)<0$ and has the same type of boundary values on the imaginary axis; the functions 
represented by these boundary values are respectively the Plancherel transforms of $f_{+}$and $f_{-}$. Putting $F_{1}=F-F_{+}, F_{1}$ is analytic in the right half-plane and has boundary values in the sense of local $L_{1}$-convergence on the imaginary axis which define the Plancherel transform of $f_{-}$. Thus the boundary values of $F_{1}$, as $x \rightarrow 0$ through positive values, agree a.e. with those of $F_{-}$as $x \rightarrow 0$ through negative values. It follows from the reflection principle that $F_{-}$is the analytic continuation of $F_{1}$. [This is stated e.g. in Titchmarsh's Theory of functions for continuous boundary values, but the proof applies equally well to the case of boundary values in the sense of local $L_{1}$-convergence.] Thus $F_{1}$ can be extended to an entire function. Now $F_{+}$is in $\mathfrak{H}_{2}(0, \infty)$, and $F_{1}$ is easily seen to satisfy the same conditions as those assumed for $F$ and hence it may now be assumed that $F$ is entire.

Let $\epsilon$ be an arbitrary positive number, and let $F_{e}(z)=\int_{0}^{e} F(z+i u) d u$. As $F_{c}$ on the imaginary axis is the convolution of the characteristic function of $(-\epsilon, 0)$ with the function whose value at $y$ is $F(i y)$, it is both square-integrable and bounded on the imaginary axis. Also $\left|F_{e}(z)\right| \leqq\left\{\int_{0}^{e} \mu+|K(z+i u)| d u\right\}$ - $\sup _{0 \leqq u \leqq \epsilon} \mid\left(P(z+i u) \mid \leqq \epsilon^{1 / 2}\left(\mu+\|K\|_{2}\right) Q(z)\right.$, with $\lim _{r \rightarrow \infty} r^{-1}|\log Q(z)|=0$ uniformly in $\theta=\arg z$, since $\lim _{r \rightarrow \infty} r /|z+i u|=1$ uniformly in $\theta$. Since $F_{c}$ is entire, it can be concluded from the Phragmen-Lindelöf theorem that $F_{\boldsymbol{c}}$ is bounded in the half-plane $R(z) \geqq 0$. Consequently $(1+\epsilon z)^{-1} F_{\mathrm{e}}(z)$ defines a function in $\mathfrak{H}_{2}(0, \infty)$, so that

$$
\int_{-\infty}^{\infty}\left|\frac{F_{\epsilon}(z)}{1+\epsilon z}\right|^{2} d y \leqq \int_{-\infty}^{\infty}\left|\frac{F_{\epsilon}(i y)}{1+\epsilon i y}\right|^{2} d y .
$$

As $\epsilon \rightarrow 0, F_{e}(i y) \rightarrow F(i y)$ in the sense of convergence in $L_{2}(-\infty, \infty)$ and $(1+\epsilon i y)^{-1}$ remains bounded and converges everywhere, from which it follows readily that the right-hand expression in the preceding inequality converges to $\int_{-\infty}^{\infty}|F(i y)|^{2} d y$. Applying Fatou's lemma to the left-hand expression it results that $\int_{-\infty}^{\infty}|F(z)|^{2} d y \leqq \int_{-\infty}^{\infty}|F(i y)|^{2} d y$.

The next lemma is essentially a special case of the extension of the preceding lemma to the case when the group $G$ is $n$-dimensional. While this complete extension could be proved along generally similar lines, the proof would require a relatively involved examination of the boundary values of functions of several complex variables which can be avoided in the present case because of the precise information available concerning the boundary values of the functions of class $\mathfrak{H}_{2}$.

LеммA 3.5. Let $\phi$ be analytic in the open tube over the first octant $C_{n}$ in $n$ dimensional space, and satisfy there the inequality $|\phi(z)| \leqq \mu e^{\|z\|^{\alpha}}, \alpha<1$.

Suppose that $F$ is in $\mathcal{H}_{2}\left(C_{n}\right)$ and that $\phi(i y) F(i y)$ is square-integrable on $G^{*}$ (i.e. on the spine of the tube). Then $\phi F$ is in $\mathfrak{H}_{2}\left(C_{n}\right)$.

For $n=1$, this follows from the preceding lemma. As the basis of an induction proof, assume the conclusion is valid when dimension $G=n-1$, and con- 
sider the case dimension $G=n$. A function $F$ in $\mathfrak{F C}_{2}\left(C_{n}^{*}\right)$ has a representation of the form

$$
F\left(z_{1}, \cdots, z_{n}\right)=\int_{C_{n}} \exp \left(-\sum_{i=1}^{n} z_{i} u_{i}\right) f\left(u_{1}, \cdots, u_{n}\right) d u,
$$

where $f \in L_{2}\left(C_{n}\right)$. From the Plancherel theory it results that $\int e^{-i y_{1} u_{1}}$ $\cdot f\left(u_{1}, \cdots, u_{n}\right) d u=g\left(y_{1}, u_{2}, \cdots, u_{n}\right)$, exists in a mean sense and is a squareintegrable function of $y_{1}, u_{2}, \cdots, u_{n}$ : By the Fubini theorem, for almost all fixed $y_{1}, g\left(y_{1}, u_{2}, \cdots, u_{n}\right)$ is square-integrable as a function of $u_{2}, \cdots, u_{n}$, so that its Laplace transform $F\left(i y_{1}, z_{2}, \cdots, z_{n}\right)$ is in $\mathcal{H C}_{2}\left(C_{n-1}^{*}\right)$, for almost all $y_{1}$. Now as $\phi F_{0} \in \mathcal{F C}_{2}\left(C_{n}^{*}\right)$, where $F_{0}(z)=\prod_{j-1}^{n} \exp \left(-z_{j}^{\beta}\right), \quad \alpha<\beta<1$, $\phi\left(i y, z_{2}, \cdots, z_{n}\right)$ can be defined for almost all $y_{1}$ in such a way that it is analytic and $\left(\phi F_{0}\right)\left(i y_{1}, z_{2}, \cdots, z_{n}\right)=\phi\left(i y_{1}, z_{2}, \cdots, z_{n}\right) F_{0}\left(i y_{1}, z_{2}, \cdots, z_{n}\right)$. It is easily verified that $\left|\phi\left(i y_{1}, z_{2}, \cdots, z_{n}\right)\right| \leqq \mu^{\prime} \exp \left\{\left\|\left(z_{2}, \cdots, z_{n}\right)\right\| \alpha\right\}$.

Now $F\left(i y_{1}, z_{2}, \cdots, z_{n}\right)$ is in $\mathcal{H}_{2}\left(C_{n-1}^{*}\right)$ for almost all $y_{1} . \phi\left(i y_{1}, z_{2}, \cdots, z_{n}\right)$ has as its boundary values on the spine of the tube over $C_{n-1}^{*}$, for almost all fixed $y_{1}, \phi\left(i y_{1}, i y_{2}, \cdots, i y_{n}\right)$, and the corresponding boundary values of $F\left(i y_{1}, z_{2}, \cdots, z_{n}\right)$ are $F\left(i y_{1}, i y_{2}, \cdots, i y_{n}\right)$. By the Fubini theorem, for almost all fixed $y_{1}, \phi\left(i y_{1}, \cdots, i y_{n}\right) F\left(i y_{1}, \cdots, i y_{n}\right)$ is square-integrable as a function of $y_{2}, \cdots, y_{n}$. Hence by the induction hypothesis, for almost all fixed $y_{1}, \phi\left(i y_{1}, z_{2}, \cdots, z_{n}\right) F\left(i y_{1}, z_{2}, \cdots, z_{n}\right)$ is in $\mathcal{H C}_{2}\left(C_{n-1}^{*}\right)$. This means in particular that

$$
\begin{aligned}
\int\left|\phi\left(i y_{1}, z_{2}, \cdots, z_{n}\right) F\left(i y_{1}, z_{2}, \cdots, z_{n}\right)\right|^{2} d y_{2}, \cdots, d y_{n} \\
\leqq \int\left|\phi\left(i y_{1}, i y_{2}, \cdots, i y_{n}\right) F\left(i y_{1}, i y_{2}, \cdots, i y_{n}\right)\right|^{2} d y_{2}, \cdots, d y_{n}
\end{aligned}
$$

for almost all $y_{1}$. Integrating both sides of this inequality with respect to $y_{1}$ and applying the Fubini theorem again, it follows that

$$
\int\left|\phi\left(i y_{1}, z_{2}, \cdots, z_{n}\right) F\left(i y_{1}, z_{2}, \cdots, z_{n}\right)\right|^{2} d y_{1}
$$

is finite for almost all $y_{2}, \cdots, y_{n}$, for fixed values of $x_{2}, \cdots, x_{n}\left(z_{i}=x_{i}+i y_{i}\right)$. From this it is easy to conclude that $\phi\left(z_{1}, z_{2}, \cdots, z_{n}\right) F\left(z_{1}, z_{2}, \cdots, z_{n}\right)$, as a function of $z_{1}$ for fixed $z_{2}, \cdots, z_{n}$, satisfies the conditions of the preceding lemma provided $\left(y_{2}, \cdots, y_{n}\right)$ is not in the proscribed null set. This implies

$$
\begin{aligned}
\int\left|\phi\left(z_{1}, z_{2}, \cdots, z_{n}\right) F\left(z_{1}, \cdots, z_{n}\right)\right|^{2} d y_{1} \\
\\
\quad \leqq \int\left|\phi\left(i y_{1}, z_{2}, \cdots, z_{n}\right) F\left(i y_{1}, z_{2}, \cdots, z_{n}\right)\right|^{2} d y_{1},
\end{aligned}
$$


again for $\left(y_{2}, \cdots, y_{n}\right)$ outside the proscribed null set. Integrating both sides of this inequality with respect to $y_{2}, \cdots, y_{n}$, it results that

$$
\begin{aligned}
\int\left|\phi\left(z_{1}, \cdots, z_{n}\right) F\left(z_{1}, \cdots, z_{n}\right)\right|^{2} d y_{1}, \cdots, d y_{n} \\
\\
\quad \leqq \int\left|\phi\left(i y_{1}, z_{2}, \cdots, z_{n}\right) F\left(i y_{1}, z_{2}, \cdots, z_{n}\right)\right|^{2} d y_{1}, \cdots, d y_{n},
\end{aligned}
$$

and integrating an earlier inequality with respect to $y_{1}$, it follows that this expression in turn is

$$
\leqq \int\left|\phi\left(i y_{1}, i y_{2}, \cdots, i y_{n}\right) F\left(i y_{1}, i y_{2}, \cdots, i y_{n}\right)\right|^{2} d y_{1}, \cdots, d y_{n},
$$

which is finite by assumption.

LeMma 3.6. The conclusion of Lemma 3.5 remains valid if $C_{n}$ is replaced by any cone spanned by $n$ (linearly independent) rays.

If $e_{1}, \cdots, e_{n}$ is any set of linearly independent vectors in $G$ and $C=\left[x: x=\sum_{i} \alpha_{i} e_{i}, \alpha_{i} \geqq 0(i=1, \cdots, n)\right]$, the transformation $f \rightarrow g$, where

$$
f\left(z_{1}, \cdots, z_{n}\right)=g\left(z_{1} e_{1}^{*}+\cdots+z_{n} e_{n}^{*}\right),
$$

$\left\{e_{1}^{*}\right\}$ being a basis dual to $\left\{e_{i}\right\}$, is easily seen to map $\mathcal{H}_{2}\left(C_{n}^{*}\right)$ onto $\mathfrak{H C}_{2}\left(C^{*}\right)$. As all the hypotheses of Lemma 3.5 are preserved under this transformation, Lemma 3.6 is a direct consequence.

LеммA 3.7. Lemma 3.5 remains valid if $C_{n}$ is replaced by an arbitrary proper cone.

If $C$ is an arbitrary proper cone, its dual contains cones $C_{0}{ }^{*}$ spanned by $n$ rays, and by the preceding lemma, $\phi F \in \mathcal{H C}_{2}\left(C_{0}^{*}\right)$, so

$$
\int|(\phi F)(z)|^{2} d y \leqq \int|(\phi F)(i y)|^{2} d y
$$

as long as $x \in C_{0}^{* 0}$. But every point in $C^{* 0}$ is contained in some $C_{0}^{* 0}$, so making use of separability, the preceding inequality holds as long as $x$ is in $C^{* 0}$.

Theorem 3 now follows at once from Lemmas 3.3 and 3.7.

5. Partial differential equations with constant coefficients. A special case of particular interest is that of the Green's operator of a linear partial differential equation with constant coefficients. The Fourier transform of such an operator is the operation of multiplication by the reciprocal of a polynomial, whose zeros form a set of measure zero, and it results that the Green's operator can thereby be formulated as a closed homogeneous operator on $L_{\mathbf{2}}(G)$. It is reasonable to expect a connection between hyperbolicity of the equation 
and causality of the Green's operator. It is also plausible that in the elliptic case there is no cone with respect to which the operator is causal. That both of those expectations are sound is indicated by the following general result, which should be compared with the work of Gårding [4] and Leray [6]. In this work there is no unique operator on a specific linear space in terms of which causality questions can be formulated, and the strict Hilbert space domains of operators do not play a role, but these authors use the Laplace transform in a spirit similar to the present use.

THEOREM 4. Let $G$ be a real vector group and $p$ a polynomial function on the dual $G^{*}$. The closed homogeneous operator on $L_{2}(G)$ whose gain is the inverse of $p$ is causal with respect to a proper cone $C$ in $G$ if and only if $p$ has no zeros in the open tube over the dual of $C$.

The "only if" part is clear from the proof of the direct part of Theorem 3 , the boundary value complications not arising because the functions involved are polynomials. To prove the "if" part, let $p$ be a polynomial with no zeros in the open tube $\Gamma^{0}$ over the dual $C^{*}$ of $C$, and let $T$ be the closed homogeneous operator on $L_{2}(G)$ whose gain is the inverse of $p(i y)$. To prove that $T$ is causal it suffices, by the arguments used in the proofs of Lemmas 3.6 and 3.7, to consider the case when $C$ is the first octant. For $\epsilon>0$, let $p_{\epsilon}$ be defined by the equation

$$
p_{\epsilon}\left(z_{1}, z_{2}, \cdots, z_{n}\right)=p\left(z_{1}+\epsilon, z_{2}+\epsilon, \cdots, z_{n}+\epsilon\right) .
$$

Then the zeros of $p_{\epsilon}$ are at a distance of at least $\epsilon$ from $\Gamma^{0}$, so that $p_{\epsilon}$ is bounded away from zero on $\Gamma^{0}$. For if $p_{\epsilon}\left(z^{(n)}\right) \rightarrow 0$ with $z^{(n)} \in \Gamma^{0}$, then clearly $\left\|z^{(n)}\right\| \rightarrow \infty$. Now for $\|z\|$ large, $p(z)$ is dominated by its principal part, and it is thereby sufficient to consider the case when $p$ is homogeneous. In this case the result is clear.

Moreover, $\sup _{z \in \Gamma}\left|p(z) / p_{c}(z)\right|$ is bounded by unity. In case $n$ (=dimension of $G)=1$, this follows by writing $p(z)=\prod_{i=1}^{k}\left(z-\alpha_{i}\right)$, so that $R\left(\alpha_{i}\right) \leqq 0$, and $p(z) / p_{\epsilon}(z)=\prod_{i=1}^{k}\left(z-\alpha_{i}\right) /\left(z+\epsilon-\alpha_{i}\right)$; now $\left(z-\alpha_{i}\right) /\left(z+\epsilon-\alpha_{i}\right)$ has for $R(z) \geqq 0$ the form $\rho /(\rho+\epsilon)$ with $R(\rho) \geqq 0$, and clearly $|\rho /(\rho+\epsilon)| \leqq 1$. For $n>1$ the result follows readily by induction, writing

$$
\frac{p(z)}{p_{\epsilon}(z)}=\frac{p\left(z_{1}, z_{2}, \cdots, z_{n}\right)}{p\left(z_{1}, z_{2}+\epsilon, \cdots, z_{n}+\epsilon\right)} \cdot \frac{p\left(z_{1}, z_{2}+\epsilon, \cdots, z_{n}+\epsilon\right)}{p\left(z_{1}+\epsilon, z_{2}+\epsilon, \cdots, z_{n}+\epsilon\right)} \cdot
$$

Now let $f$ be in $\mathcal{D}_{T} \cap L_{2}(C)$; it must be shown that $T f \in L_{2}(C)$. Putting $F$ for the Laplace transform of $f$, this is equivalent to showing that if $F(i y) / p(i y)$ is square integrable over $G$, then $F(z) / p(z) \in \mathfrak{H}_{2}(C)$. Now as $p_{\epsilon}(z)$ is bounded away from zero for $z$ in $\Gamma^{0}, F(z) / p_{\epsilon}(z)$ is in $\mathfrak{H}_{2}(C)$, so that

$$
\int\left|F(z) / p_{\epsilon}(z)\right|^{2} d y \leqq \int\left|F(i y) / p_{\epsilon}(i y)\right|^{2} d y .
$$


As $\epsilon \rightarrow 0$, the right-hand side of this inequality converges, by the Lebesgue dominated convergence theorem and the boundedness of $p(i y) / p_{c}(i y)$, to $\int|F(i y) / p(i y)|{ }^{2} d y$. Applying the Fatou lemma to the right-hand side now shows that

$$
\int|F(z) / p(z)|^{2} d y \leqq \int|F(i y) / p(i y)|^{2} d y .
$$

CoRollary 4.1. The Green's operator of the wave equation is causal with respect to any of the corresponding light cones. The Green's operator of the Laplace equation is causal with respect to no proper cone.

This is a matter of verifying the freedom from zeros and the presence of zeros, respectively, for the corresponding second degree polynomials, in the appropriate cones.

6. Domain of dependence of a continuous system. In this section a notion of domain of dependence for continuous homogeneous operators on $L_{2}(G)$ is treated. Such a domain is roughly a set $D$ in $G$ such that the value of the output at the point $x$ of $G$ depends only on the input at the points $x-a, a \in D$, and which moreover is minimal with respect to this property. The main result of this section is the existence of such a minimal set, when suitable topological and convexity restrictions are imposed on the sets involved, and the uniqueness of this minimal set. Somewhat stronger results could be obtained by using the spectral resolution for ideals in $L_{1}$ given by one of the present authors, along lines due in part to A. Calderon, but it seems of interest to develop certain completely different techniques not involving the theory of normed algebras.

Another formulation of the notion of domain of dependence is due to Leray, and involves some additional elements of structure. Its Hilbert space properties and in particular its connection with the present domain are not yet established.

A nucleus for a homogeneous operator $T$ in $L_{2}(G, K)$ is a closed convex set $N$ in $G$ with the property that if $f$ is in the domain of $T$ and vanishes outside the compact set $A$, then $T f$ vanishes outside $A \oplus N$. A domain of dependence for $T$ is defined as a minimal nucleus (if such exists).

ExAmple. Let $f$ be integrable over $G$ and let $T$ be the operator on $L_{2}(G)$ of convolution with $f$. Thus $T$ is a continuous homogeneous operator. If $f$ vanishes a.e. outside the set $P$, then it is easily seen that the closed convex hull $N$ of $P$ is a nucleus for $T$. By the separability of $G$ and the fact that a countable union of null sets is a null set, there is a minimal set $N_{0}$ arising from sets $P$ in this fashion. If $f$ is non-negative, it can be shown that furthermore $N_{0}$ is a domain of dependence and is contained in every nucleus for $T$. This result follows from the alternative characterization of $P$ as the intersection of all closed half spaces outside of which $f$ vanishes a.e. 
TheOREM 5. A continuous homogeneous operator $T$ on $L_{2}(G)$ has a unique domain of dependence, which is contained in every nucleus.

The proof depends mainly on the consideration of the nuclear half-spaces of $T$.

Lemma 5.1. A half-space $H$ whose boundary contains the origin is a nucleus for $T$ if and only if $T$ is causal with respect to $-H$.

To prove the "only if" part is to show that, given that $H$ is a nucleus, then $T f$ vanishes outside $H$. Now any element $f$ of $L_{2}(H)$ is the limit of a sequence $\left\{f_{n}\right\}$ of elements of $L_{2}(H)$ which vanish outside compact subsets $C_{n}$ of $H$, and $T f$ is then the limit of $\left\{T f_{n}\right\}$. As $H$ is nuclear for $T, T f_{n}$ vanishes outside $C_{n} \oplus H$, and $C_{n} \oplus H \subset H \oplus H=H$. Thus each $T f_{n}$, and hence the limit $T f$, vanishes outside $H$.

To prove the "if" part, it suffices to show that if $T$ is causal with respect to $H$, then for any integrable $f$ in $L_{2}(G), T f$ is in the span (in $L_{2}(G)$ ) of the $U(a) f$ with $a$ in $H$, where $(U(a) f)(x)=f(x-a)$. For it follows from this that if $f$ vanishes outside the measurable set $A$ of finite measure, then $T f$ vanishes outside $A \oplus H$.

Let $g$ be an element of $L_{2}(H)$ whose Fourier transform is bounded, so that the operation $S$ in $L_{2}(G)$ of convolution by $g$ is everywhere defined and continuous. Taking $g$ to be proportional to the characteristic function of $H \cap U$, where $U$ is an open sphere around the origin, it is readily seen that $T f$ is a limit of functions of the form TSf. It therefore suffices to show that TSf is in the span of the translates of $f$ by elements of $H$. Now $T S f=T(g * f)=h * f$, where $h=T g$. As $T$ is causal, $h$ is in $L_{2}(H)$. As the basis for an indirect proof, assume now that $h * f$ is not in the span of the $U(a) f, a \in H$. Then there exists an element $k$ of $L_{2}(G)$ that is orthogonal to all the $U(a) f, a \in H$, but not to $h * f$.

The operation of convolation (of elements of $L_{2}(G)$ ) by the integrable function $f$ is continuous and has as its adjoint the operation of convolution by $f^{\sim}$, where $f^{\sim}(x)=\bar{f}(-x)$. Therefore $(h * f, k)=\left(h, f^{\sim} * k\right)$.

It is easily verified that $\left(f^{\sim} * k\right)(a)$ is the complex conjugate of $(U(a) f, k)$, which shows that $\left(f_{*} * k\right)(a)=0$ for $a$ in $H$. On the other hand, $h(a)=0$ for $a$ not in $H$, as $h \in L_{2}(H)$. It results that $\left(h, f^{\sim} * k\right)=0$, yielding the contradiction that $k$ is not orthogonal to $f^{\sim * k}$.

Proof of theorem. Let $D$ be the intersection of all nuclear half-spaces of the continuous homogeneous operator $T$. If $N$ is a nucleus, the same is true of any half-space containing $N$, and as $N$ is the intersection of such halfspaces, $D$ is contained in every nucleus. To establish the theorem it therefore suffices to show that $D$ is itself a nucleus.

For any element $u$ of $G^{*}$, let $\omega(u)$ be the g.l.b. of all extended numbers $\alpha($ real or $+\infty)$ for which the half-space $H=[x: u(x) \leqq \alpha]$ is nuclear. Then the 
half-space $H_{n}=[x: u(x) \leqq \omega(u)]$ is likewise nuclear, whether proper (i.e. distinct from $G$ and also from the empty set) or otherwise. In case $H_{u}$ is proper, this is true because every $\epsilon$-neighborhood of $H_{u}$ is a nuclear half-space, and whenever a closed convex set $K$ has the property that $K \oplus S_{\text {e }}$ is nuclear for all $\epsilon$-spheres $S_{\epsilon}$ around the origin, then $K$ is itself nuclear. To see this last fact, note that if $A$ is compact, then $A \oplus K$ is closed, so that

$$
\bigcap_{\epsilon>0}\left(A \oplus K \oplus S_{\mathrm{\epsilon}}\right)=A \oplus K \text {. }
$$

In case $H_{u}=G$, the conclusion is trivial. The only remaining alternative is that $H_{u}$ is empty, and in this case the conclusion follows from the fact that in this case $T=0$. To prove this it suffices to consider the case when $u\left(x_{1}, \cdots, x_{n}\right)=x_{1}$.

If $f\left(x_{1}, \cdots, x_{n}\right)$ is in $L_{2}(G)$, then for almost all $x_{1}, f\left(x_{2}, \cdots, x_{n}\right)$ is in $K=L_{2}\left(R^{n-1}\right)$, where $R$ is the additive group of the reals, and defining $f^{\prime}$ on $R$ by the equation

$$
f^{\prime}\left(x_{1}\right)=f\left(x_{1}, \cdots\right),
$$

then the correspondence $f \rightarrow f^{\prime}$ is unitary on $L_{2}(G)$ to $L_{2}(R, K)$. The operator $T$ is carried thereby into a continuous homogeneous operator $T^{\prime}$ on $L_{2}(R, K)$, and the nuclear property for the half-spaces $\left[x: x_{1} \leqq \alpha\right]$, relative to the operator $T$ on $L_{2}(G)$, implies the same property for the rays $\left[x_{1}: x_{1} \leqq \alpha\right]$, relative to the operator $T^{\prime}$ on $L_{2}(R, K)$. This in turn implies that $(-\infty, 0)$ is nuclear for $V(\alpha) T^{\prime}$, where $V(\alpha)$ denotes the operation of translation through $-\alpha$. It follows readily from Lemma 5.1 that $(0, \infty)$ is causal for $V(\alpha) T^{\prime}$, the only point being that the lemma is valid for $K$-valued inputs as well as for complex-valued ones, this fact following by a slight variation of the proof of the "only if" part of the lemma. The extension of the gain of $V(\alpha) T^{\prime}$ to the right half-plane is therefore bounded by $\left\|V(\alpha) T^{\prime}\right\|=\left\|T^{\prime}\right\|$. On the other hand if $\phi$ is the gain of $T^{\prime}$, then $e^{-\alpha i \nu} \phi(i y)$ is that of $V(\alpha) T^{\prime}$, so that $\left|e^{-\alpha \dot{x}} \phi(z)\right| \leqq\|T\|$ for $x>0$ and arbitrary real $\alpha$. It follows, letting $\alpha \rightarrow-\infty$, that $\phi=0$, so that $T=0$.

Now $\omega$ has the following properties: (1) $\omega(\gamma u)=\gamma \omega(u)$ if $\gamma \geqq 0$; (2) $\omega(u+v)$ $\leqq \omega(u)+\omega(v)$. Property (1) can be verified directly. To verify (2), let $a$ be a common point of the boundaries of $H_{u}$ and $H_{v}$, assuming for the moment that $H_{u}$ and $H_{v}$ are proper half spaces. Then both $H_{u} \ominus a$ and $H_{v} \ominus a$ are nuclear for $U(a) T$. By Lemma 5.1, this means that $U(a) T$ is causal with respect to both $H_{u} \ominus a$ and $H_{v} \ominus a$. Now if a continuous homogeneous operator is causal with respect to two cones, it is causal also with respect to their intersection. In particular, $U(a) T$ is causal with respect to $\left(H_{u} \ominus a\right) \cap\left(H_{v} \ominus a\right)$ $=\left(H_{u} \cap H_{v}\right) \ominus a$. The proof of Lemma 5.1 shows that if a continuous homogenous operator is causal with respect to a cone, then the cone is a nucleus for the operator. Therefore, $\left(H_{u} \cap H_{v}\right) \ominus a$ is nuclear for $U(a) T$, which means 
that $H_{u} \cap H_{v}$ is nuclear for $T$. Now in case one or both of $H_{u}$ and $H_{v}$ is an improper half-space, it is trivial that $H_{u} \cap H_{v}$ is nuclear. Thus, in any case the intersection of two nuclear half spaces is nuclear. Now it is clear that

$$
[x:(u+v)(x) \leqq \omega(u)+\omega(v)] \supset[x: u(x) \leqq \omega(u)] \cap[x: v(x) \leqq \omega(v)] .
$$

As the set on the right-hand side of this inclusion relation is nuclear, so also is the set on the left-hand side, which means that $\omega(u+v) \leqq \omega(u)+\omega(v)$.

From the definition of $D, D=\bigcap_{u}[x: u(x) \leqq \omega(u)]$, and as $\omega$ satisfies (1) and (2), $\omega$ is the support function for $D$. For any $\epsilon>0, \cap_{u}\left(H_{u} \oplus K_{\epsilon}\right)$ is the convex set $[x: u(x) \leqq \omega(u)+\epsilon\|u\|]$, and as $\omega(u)+\epsilon\|u\|$ is a convex function of $u$, it is the support function of this set. On the other hand, it is well known to be the support function of $D \oplus K_{\epsilon}$, and it results that $\cap_{u}\left(H_{u} \oplus K_{\epsilon}\right)=D \oplus K_{\epsilon}$.

Now let $\left\{g_{n}\right\}$ be a sequence of non-negative elements of $L_{2}(G)$ such that $g_{n}$ vanishes outside $K_{n}$ and $\int g_{n}=1$. Then for any $f$ in $L_{2}(G), g_{n} * f \rightarrow f$ in $L_{2}(G)$. Now $T f=\lim _{n} T\left(g_{n} * f\right)=\lim _{n} T g_{n} * f$. For any $u$ in $G^{*}, T g_{n}$ vanishes outside $H_{u} \oplus K_{\epsilon}$, with $\epsilon=n^{-1}$, so that it vanishes also outside $\bigcap_{u}\left(H_{u} \oplus K_{\epsilon}\right)=D \oplus K_{\epsilon}$. It results that if $f$ vanishes outside the compact set $A$, then $T g_{n} * f$ vanishes outside $A \oplus D \oplus K_{\text {e. Hence }} T f$ vanishes outside $A \oplus D \oplus K_{\ell}$, for each $\epsilon$, and therefore also outside $A \oplus D$, by the same argument as in a similar situation above.

The argument above shows that if $f$ is integrable, then $T f$ is in the span of the translates of $f$ by the elements of $A \oplus D \oplus K_{\epsilon}$, for any $\epsilon>0$. It is not clear whether this remains valid when $f$ is arbitrary in $L_{2}(G)$, or when $\epsilon=0$. This holds however in an interesting special case.

Corollary 5.1. If the domain of dependence of the bounded homogeneous operator $T$ consists only of the point $\alpha$, then $T$ is translation by $\alpha$, within multiplication by a nonzero scalar.

It suffices to take the case $\alpha=0$. In this case the gain of $T$ admits bounded analytic extensions to the tubes over both the right and the left-hand spaces, $x_{1}>0$ and $x_{1}<0$. By the reflection principle, these extensions form a single analytic function. As it is bounded throughout $G \oplus i G$, it is, by Liouville's theorem, a constant.

\section{REFERENCES}

1. S. Bochner, Bounded analytic functions in several complex variables and multiple Laplace integrals, Amer. J. Math. vol. 59 (1937) pp. 732-738.

2. - Group invariance of Cauchy's formula in several variables, Ann. of Math. vol. 45 (1944) pp. 686-707.

3. S. Bochner and W. T. Martin, Several complex variables, Princeton, 1948.

4. L. Gårding, Linear hyperbolic partial differential equations with constant coefficients, Acta Math. vol. 85 (1951) pp. 1-62.

5. Jean Leray, Symbolic calculus for several variables and boundary value problems for differential equations, Lectures at the Institute for Advanced Study, 1951-52. 
6. - Les solutions elementaires d'une equation aux dérives partielles, d coefficients constants, C. R. Acad. Sci. Paris vol. 234 (1952) pp. 1112-1115.

7. R. E. A. C. Paley and N. Wiener, Fourier transforms in the complex domain, Amer. Math. Soc. Colloquium Publications, vol. 19, 1934.

8. I. E. Segal, $A$ noncommutative extension of abstract integration, Ann. of Math. vol. 57 (1953) pp. 401-457.

9. N. Wiener, Extrapolation, interpolation, and smoothing of stationary time series, with engineering applications, Massachusetts Institute of Technology, 1949.

Institute for Advanced Study,

Princeton, N. J.

UNIVERSity OF MARSEILles,

Marsemles, France.

University of Chicago,

Chicago, Ill. 